\title{
Scintillating bolometric technique for the neutrino-less double beta decay search: The LUCIFER/CUPID-0 experiment
}

\author{
N. Casali ${ }^{\mathrm{a}, \mathrm{c}, *}$, D.R. Artusa ${ }^{\mathrm{b}, \mathrm{f}}, \mathrm{F}$. Bellini $^{\mathrm{a}, \mathrm{c}}$, M. Biassoni $^{\mathrm{d}, \mathrm{h}}$, \\ C. Rusconi ${ }^{\text {h }}$, K. Schäffner ${ }^{\mathrm{k}}$, C. Tomei $^{\mathrm{c}}$, M. Vignati $^{\mathrm{c}}$ \\ a Dipartimento di Fisica, Sapienza Università di Roma, Roma I-00185, Italy \\ b Department of Physics and Astronomy, University of South Carolina, Columbia, SC 29208, USA \\ c INFN - Sezione di Roma, Roma I-00185, Italy \\ d Dipartimento di Fisica, Università di Milano-Bicocca, Milano I-20126, Italy \\ e INFN - Laboratori Nazionali di Legnaro, Legnaro (Padova) I-35020, Italy \\ ${ }^{\mathrm{f}}$ INFN - Laboratori Nazionali del Gran Sasso, Assergi (L'Aquila) I-67010, Italy \\ g INFN - Sezione di Genova, Genova I-16146, Italy \\ h INFN - Sezione di Milano Bicocca, Milano I-20126, Italy \\ ${ }^{\text {i }}$ Dipartimento di Fisica, Università di Genova, Genova I-16146, Italy \\ ${ }^{j}$ Physics Department - Princeton University, Washington Road, 08544 Princeton, NJ, USA \\ ${ }^{\mathrm{k}}$ INFN - Gran Sasso Science Institute, 67100 L'Aquila, Italy
}

A. Camacho ${ }^{\mathrm{e}}$, S. Capelli ${ }^{\mathrm{d}, \mathrm{h}}$, L. Cardani ${ }^{\mathrm{a}, \mathrm{c}, \mathrm{j}}$, P. Carniti ${ }^{\mathrm{d}, \mathrm{h}}$, L. Cassina ${ }^{\mathrm{d}, \mathrm{h}}$, M. Clemenza ${ }^{\mathrm{d}, \mathrm{h}}$, O. Cremonesi ${ }^{\text {h }}$, A. Cruciani ${ }^{\mathrm{a}, \mathrm{c}}$, A. D'Addabbo ${ }^{\mathrm{f}}$, I. Dafinei ${ }^{\mathrm{c}}$, S. Di Domizio ${ }^{\mathrm{g}, \mathrm{i}}$, M.L. di Vacri ${ }^{\mathrm{f}}$, F. Ferroni ${ }^{\mathrm{a}, \mathrm{c}}$, L. Gironi ${ }^{\mathrm{d}, \mathrm{h}}$, C. Gotti $^{\mathrm{d}, \mathrm{h}}$, G. Keppel ${ }^{\mathrm{e}}$, M. Maino ${ }^{\mathrm{d}, \mathrm{h}}$, M. Martinez $^{\mathrm{a}, \mathrm{c}}$, , S. Morganti $^{\mathrm{c}}$, S. Nagorny ${ }^{k}$, D. Orlandi ${ }^{f}$, L. Pagnanini ${ }^{k}$, M. Pallavicini ${ }^{g, i}$, V. Palmieri ${ }^{e}$, L. Pattavina ${ }^{f}$, M. Pavan ${ }^{\mathrm{d}, \mathrm{h}}$, G. Pessina ${ }^{\mathrm{h}}$, V. Pettinacci ${ }^{\mathrm{a}, \mathrm{c}}$, S. Pozzi $^{\mathrm{d}, \mathrm{h}}$, S. Pirro ${ }^{\mathrm{f}}$, E. Previtali ${ }^{\mathrm{h}}$, A. Puiu $^{\mathrm{d}, \mathrm{h}}$,

\section{A R T I C L E I N F O}

Article history:

Received 21 March 2016

Accepted 13 June 2016

Available online 16 June 2016

Keywords:

Scintillating bolometers

Enriched crystal

Neutrino-less double beta decay

Particle identification

\begin{abstract}
A B S T R A C T
CUPID is a proposed future tonne-scale bolometric neutrino-less double beta decay $(0 \nu \beta \beta)$ experiment to probe the Majorana nature of neutrinos and discover lepton number violation in the so-called inverted hierarchy region of the neutrino mass. In order to improve the sensitivity with respect to the current bolometric experiments, the source mass must be increased and the backgrounds in the region of interest must be dramatically reduced. The background suppression can be achieved discriminating $\beta / \gamma$ against $\alpha$ events by means of the different light yield produced in the interactions within a scintillating bolometer. The increase in the number of $0 \nu \beta \beta$ emitters demands for crystals grown with enriched material. LUCIFER/CUPID-0, the first demonstrator of CUPID, aims at running the first array of enriched scintillating $\mathrm{Zn}^{82} \mathrm{Se}$ bolometers (total mass of about $7 \mathrm{~kg}$ of ${ }^{82} \mathrm{Se}$ ) with a background level as low as $10^{-3}$ counts $/(\mathrm{keV} \mathrm{kg} \mathrm{y})$ in the energy region of interest. We present the results of the first measurement performed on three $\mathrm{Zn}^{82}$ Se enriched scintillating bolometers operated deep underground in the Hall $\mathrm{C}$ of the Laboratori Nazionali del Gran Sasso.
\end{abstract}

(c) 2016 Elsevier B.V. All rights reserved.

\section{Introduction}

The most sensitive bolometric experiment searching for neutrino-less double beta decay $\left(\begin{array}{ll}0 & \nu \beta \beta\end{array}\right)$ will be CUORE (Cryogenic Underground Observatory for Rare Events) [1] located in the Laboratori Nazionali del Gran Sasso (LNGS). With an array of

\footnotetext{
* Corresponding author at: Dipartimento di Fisica, Sapienza Università di Roma, Roma I-00185, Italy.

E-mail address: nicola.casali@roma1.infn.it (N. Casali).
}

988 $\mathrm{TeO}_{2}$ bolometers $(5 \times 5 \times 5 \mathrm{~cm})^{3}$ corresponding to $206 \mathrm{~kg}$ of ${ }^{130} \mathrm{Te}$, and a background level of 0.01 counts/(keV kg y) CUORE will be able to reach a sensitivity on the effective Majorana mass of neutrino $\left(m_{\beta \beta}\right)$ of about $(0.05 \div 0.13) \mathrm{eV}$, which represent the beginning of the inverted hierarchy region of the neutrino mass. The main background in the energy region of interest (about $2.5 \mathrm{MeV}$ ) will come from $\alpha$ particles produced by surface contaminations in ${ }^{238} \mathrm{U}$ and ${ }^{232} \mathrm{Th}$ (and their daughters) on the crystals and on the copper structure that supports the array. In addition, since the Q-value of the ${ }^{130} \mathrm{Te} 0 \nu \beta \beta$ decay [2] lies between the ${ }^{208} \mathrm{Tl} \gamma$ line at $2615 \mathrm{keV}$ and its Compton edge, also multiCompton events 
contribute to the background budget of the experiment.

Next generation $0 \nu \beta \beta$ experiments [3] aim to reach a sensitivity on $m_{\beta \beta}$ at a level of $0.01 \mathrm{eV}$, in order to completely explore the inverted hierarchy region.

To reach this ambitious goal the source mass must be increased and the background in the region of interest dramatically reduced. To increase the number of $0 \nu \beta \beta$ emitters crystals grown with enriched material are needed. The background suppression can be achieved rejecting the $\alpha$ interactions by performing a particle identification and simultaneously choosing isotopes with a Q-value above the $2615 \mathrm{keV}$ line $\left({ }^{208} \mathrm{Tl}\right)$.

The CUORE Upgrade with Particle IDentification (CUPID) collaboration aims to run the first demonstrator of a future inverted hierarchy explorer, operating 30 crystals of $\mathrm{Zn}^{82} \mathrm{Se}$ as scintillating bolometer. This detector, named LUCIFER/CUPID-0, will study the $0 \nu \beta \beta$ of ${ }^{82} \mathrm{Se}$ which is considered one of the most interesting candidates because of its high Q-value, $2998 \mathrm{keV}$ [4]. Se was enriched up to $95.4 \%$ in order to overcome the rather low isotopic abundance of ${ }^{82} \mathrm{Se}(8.73 \%)$. The ${ }^{82} \mathrm{Se}$ was embedded in $440 \mathrm{~g} \mathrm{ZnSe}$ crystals that can be operated as scintillating bolometers [5]. A scintillating bolometer is a crystal that, beside working as calorimeter at cryogenic temperatures, also emits scintillation light; the light yield (LY) produced in the particle interaction is strongly dependent on the particle itself: for a fixed energy released in the bolometer the LY of a $\beta / \gamma$ particle is very different from an $\alpha$ one allowing an active background rejection. To detect the emitted light, an additional detector must be faced to the main bolometer: this light detector is also a bolometer, consisting of a thin germanium wafer as absorber and a thermal sensor of the same type as the one used for the main bolometer [6]

With a total mass of about $7 \mathrm{~kg}$ of ${ }^{82} \mathrm{Se}$ and an expected background level of about $10^{-3}$ counts/( $\mathrm{keV} \mathrm{kg} \mathrm{y)} \mathrm{the} \mathrm{CUPID-0} \mathrm{detector}$ will be able to reach a sensitivity of the same order of magnitude of CUORE, despite it is 56 times smaller in terms of detector mass and 30 times smaller in terms of $0 \nu \beta \beta$ emitters [7].

In the following sections we describe the CUPID-0 detector, and the results of the test performed on the first three $\mathrm{Zn}^{82} \mathrm{Se}$ bolometers.

\section{CUPID-0 detector}

The CUPID-0 detector will be composed by five towers, each hosting six $\mathrm{Zn}^{82} \mathrm{Se}$, and being surrounded by $3 \mathrm{M}$ Vikuti reflective foils to increase the light collection efficiency. Each crystal will be monitored by two germanium light detectors [6] (Ge-LD). The 30 $\mathrm{Zn}^{82}$ Se crystals are cylinders $5.5 \mathrm{~cm}$ in height and $4.4 \mathrm{~cm}$ in diameter and the $36 \mathrm{Ge}-\mathrm{LD}$ are germanium disks with $4.4 \mathrm{~cm}$ in diameter and $170 \mu \mathrm{m}$ thickness; a $\mathrm{SiO}_{2}$ layer is deposited on one face of the germanium disk to increase the light absorption.

Both $\mathrm{Zn}^{82} \mathrm{Se}$ and Ge-LD will be equipped with a NTD Ge thermistor to detect their temperature variations and a heater, a simple resistor that produces a fixed pulse at constant time intervals in the detector, allowing for stability checks. Both the sensors and the heaters will be glued onto the crystal and the light absorber using the Araldit Rapid epoxy. Both $\mathrm{Zn}^{82}$ Se bolometer and Ge-LD will be enclosed in an NOSV tough pitch copper structure, used as mechanical support as well as thermal bath for

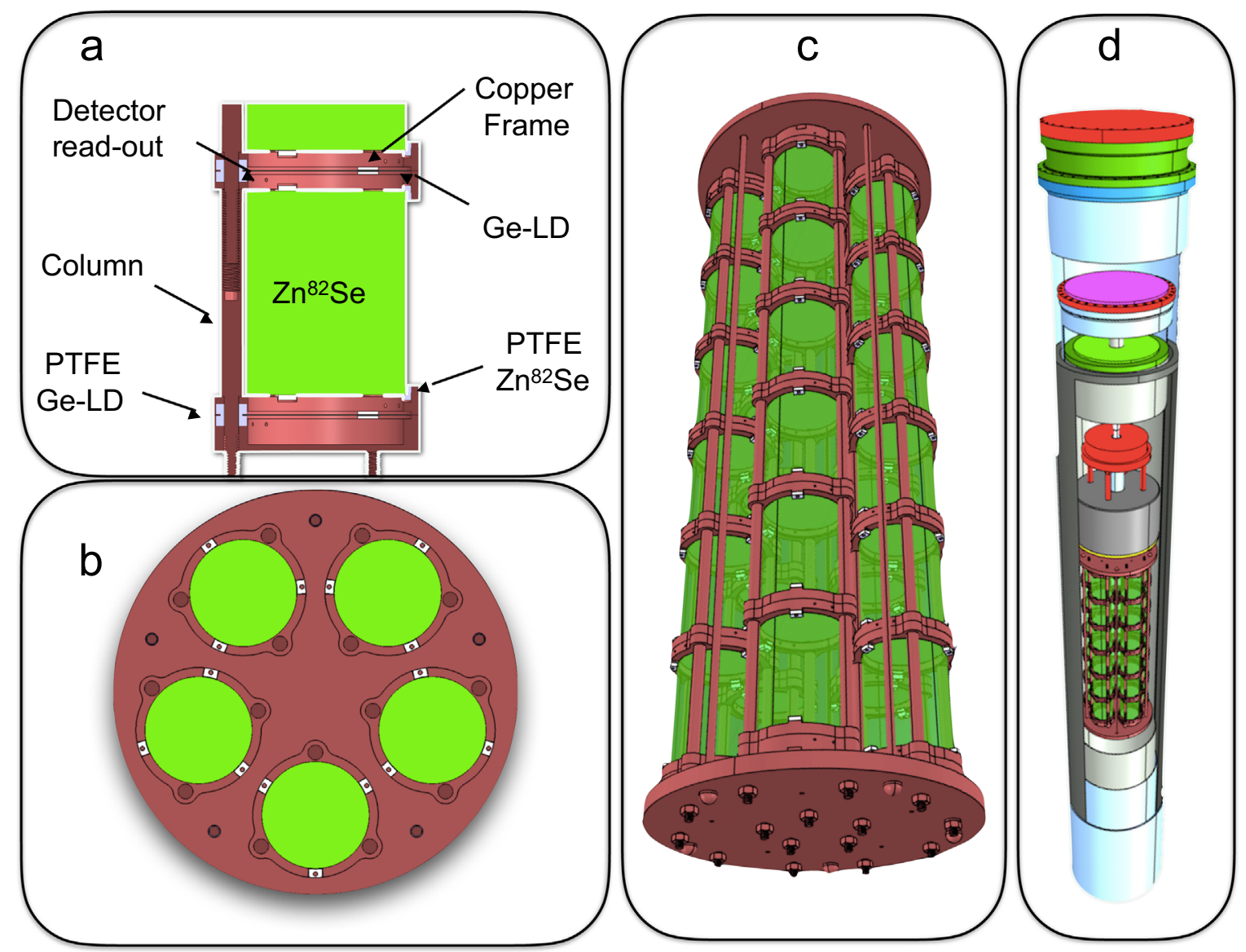

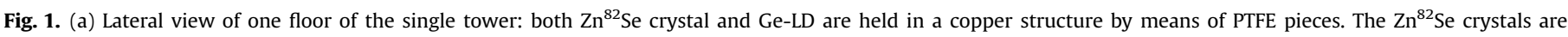

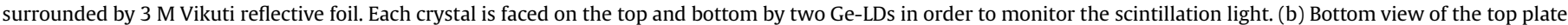
where the five towers are anchored. (c) 3D view of the CUPID-0 detector. (d) 3D view of the Hall A dilution unit with the CUPID-0 detector inside. 
the detector operation. The weak thermal conductance between absorber and heat bath will be realized by means of small PTFE pieces that, in addition, could compensate for the different thermal contractions of detectors and copper. A complete overview of the detector layout is shown in Fig. 1a,b and c. The CUDIP-0 detector will be hosted in the same cryostat used for CUORICINO [8] and CUORE-0 [9] detectors (see Fig. 1d) located deep underground in the Hall A of LNGS. For this reasons the $\gamma$ background is well known, and it is expected to be of the order of $10^{-3}$ counts/ ( $\mathrm{keV} \mathrm{kg} \mathrm{y)} \mathrm{at} \mathrm{the} 0 \nu \beta \beta$ Q-value. All the assembly line of the detector will be performed in a radon free clean room located in the Hall C of LNGS, in order to avoid radon radioactive contaminations of the detector [10].

The CUPID-0 detector is expected to start operation in the autumn 2016.

\section{Experimental set-up of the first $\mathrm{Zn}^{82} \mathrm{Se}$ test}

The first three enriched $\mathrm{Zn}^{82}$ Se bolometers, each one equipped with two Ge-LD are assembled using the same detector design shown in Fig. 1a and anchored to the mixing chamber of the CUORE/LUCIFER R\&D dilution refrigerator located in the Hall $C$ of LNGS [11]. The read-out of the thermistors is performed using the Cuoricino electronics [12]. The voltage signals, amplified and filtered by means of an anti-aliasing 6-pole active Bessel filter (120 dB/decade), were acquired by a NI PXI-6284 18-bits ADC with a sampling frequency of $1 \mathrm{kHz}$ for the $\mathrm{Zn}^{82}$ Se crystals and $2 \mathrm{kHz}$ for the Ge-LDs. The Bessel cut-off frequency could be adjusted according to the signal bandwidth and to the noise contributions. For these test, the Bessel cut-off was set at $200 \mathrm{~Hz}$ for the Ge-LDs and at $63 \mathrm{~Hz}$ for the $\mathrm{Zn}^{82} \mathrm{Se}$. Further details on the cryogenic facility and the electronic read-out can be found in Refs. [11-13]. The trigger is software generated on each bolometer: if the amplitude of a $\mathrm{Zn}^{82}$ Se pulse or a Ge-LD pulse exceeds a given threshold for a given amount of time, waveforms $5 \mathrm{~s}$ long on the $\mathrm{Zn}^{82} \mathrm{Se}$ and
$250 \mathrm{~ms}$ long on the Ge-LD are saved on disk. In addition, whenever a $\mathrm{Zn}^{82} \mathrm{Se}$ pulse is triggered, a $250 \mathrm{~ms}$ window length is acquired on the two Ge-LDs too, irrespective of their triggers.

To maximize the signal to noise ratio, the waveforms are processed off-line with the optimum filter algorithm $[14,15] . \mathrm{Zn}^{82} \mathrm{Se}$ pulses are identified with a peak finder algorithm, and their amplitude is computed as the peak maximum. The pulse amplitude of Ge-LDs is evaluated exploiting the characteristics time-delay of the light signal with respect to the $\mathrm{Zn}^{82}$ Se heat pulse, which is estimated in calibration runs using events generated by particles interacting in both detectors (for more details on the algorithm see Ref. [16]).

To calibrate the $\mathrm{Zn}^{82}$ Se bolometers and to generate events near the $0 \nu \beta \beta$ region, the setup was exposed to a ${ }^{232} \mathrm{Th} \gamma$-source placed temporarily outside of the cryostat. To evaluate the discrimination power between $\beta / \gamma$ and $\alpha$ events in the energy region of interest (around the Q-value for the $0 \nu \beta \beta$ of ${ }^{82} \mathrm{Se}$ ), an $\alpha$-source was permanently placed close to the $\mathrm{Zn}^{82} \mathrm{Se}$ crystal. The source consisted in a Samarium based solution, covered with a thin mylar foil to absorb a part of the $\alpha$ s energy and thus produce a continuum spectrum in the range $0.5-2.3 \mathrm{MeV}$. The Ge-LDs are exposed to a permanent ${ }^{55} \mathrm{Fe}$ source, providing 5.9 and $6.4 \mathrm{keV}$ X-rays for a direct energy calibration.

\section{Results}

The energy resolution computed on the $\gamma$ peaks produced by the ${ }^{232} \mathrm{Th}$ calibration source is reported in Fig. 2-Left for the $\mathrm{Zn}^{82} \mathrm{Se}-01$.

Fitting with a line this energy trend we are able to extrapolate an energy resolution at the $0 \nu \beta \beta$ energy that results about $30 \mathrm{keV}$ FWHM. The remaining two $\mathrm{Zn}^{82} \mathrm{Se}$ crystals showed consistent results.

The ${ }^{55} \mathrm{Fe}$ spectrum acquired by one of the four Ge-LDs is shown in Fig. 2-Right: the two peaks at 5.9 and $6.4 \mathrm{keV}$ are fitted using
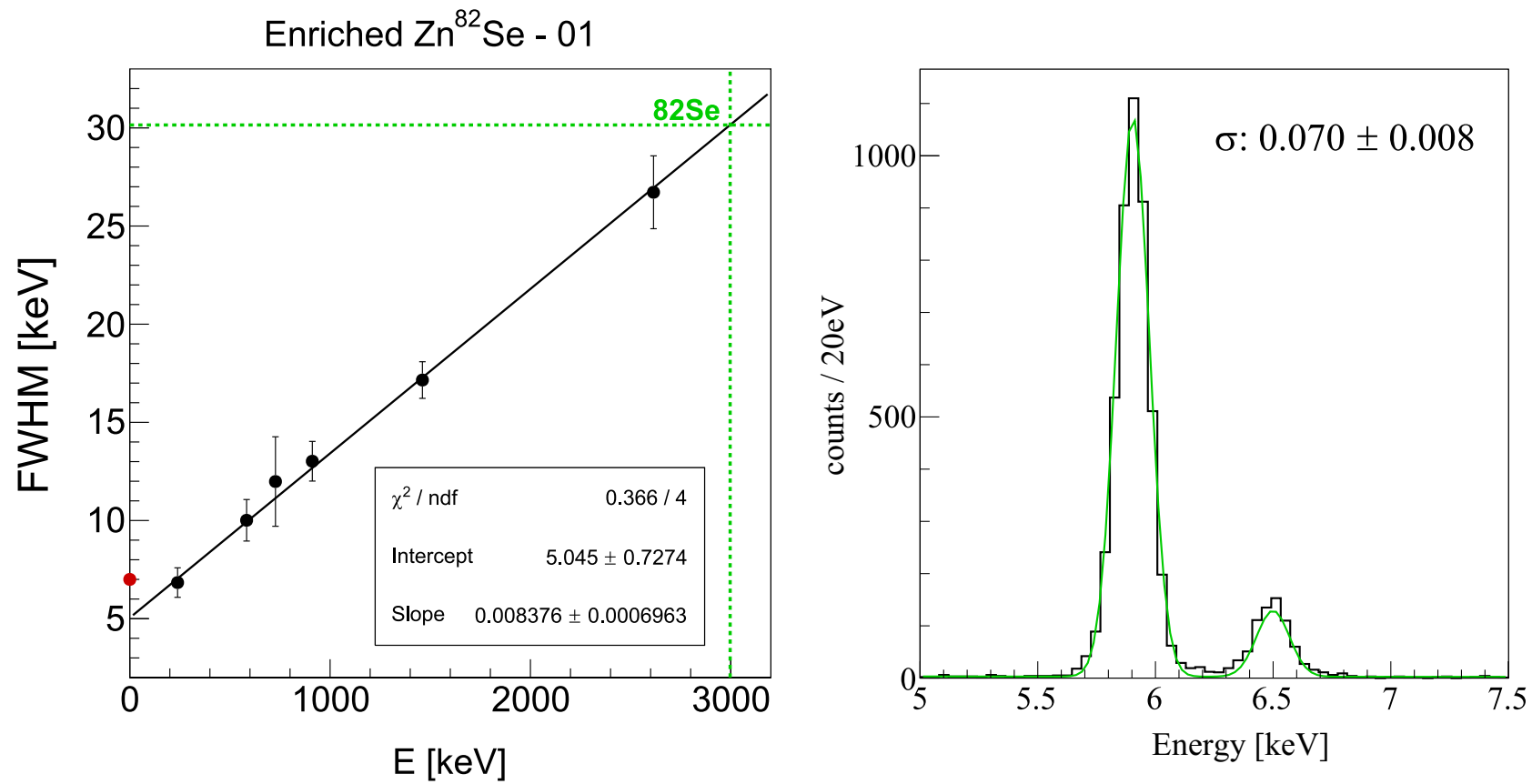

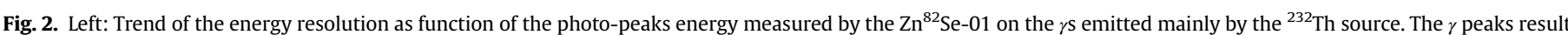

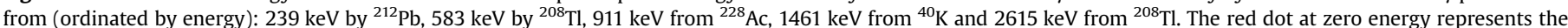

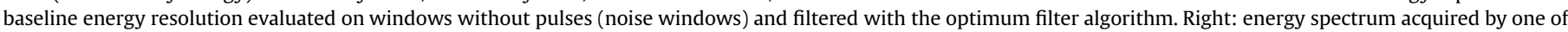

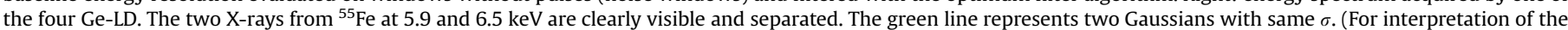
references to color in this figure legend, the reader is referred to the web version of this article.) 


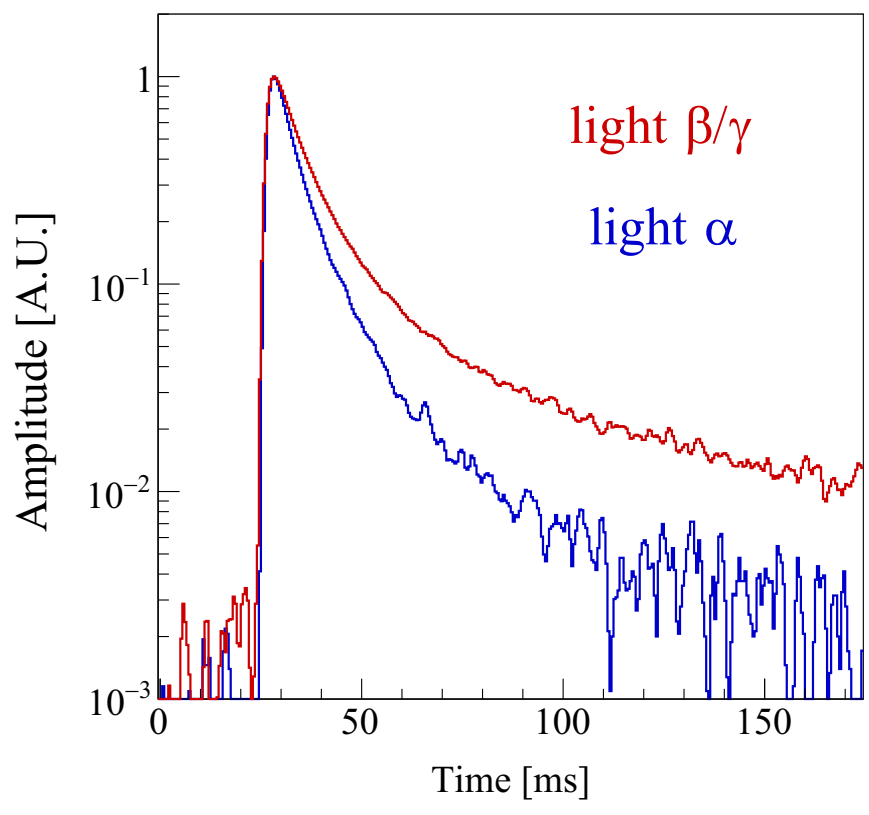

Fig. 3. Average light pulses for $\beta / \gamma$ interactions (red line) and $\alpha$ ones (blue line). The slower decay time for the $\beta / \gamma$ light signal is clearly visible. (For interpretation of the references to color in this figure legend, the reader is referred to the web version of this article.)

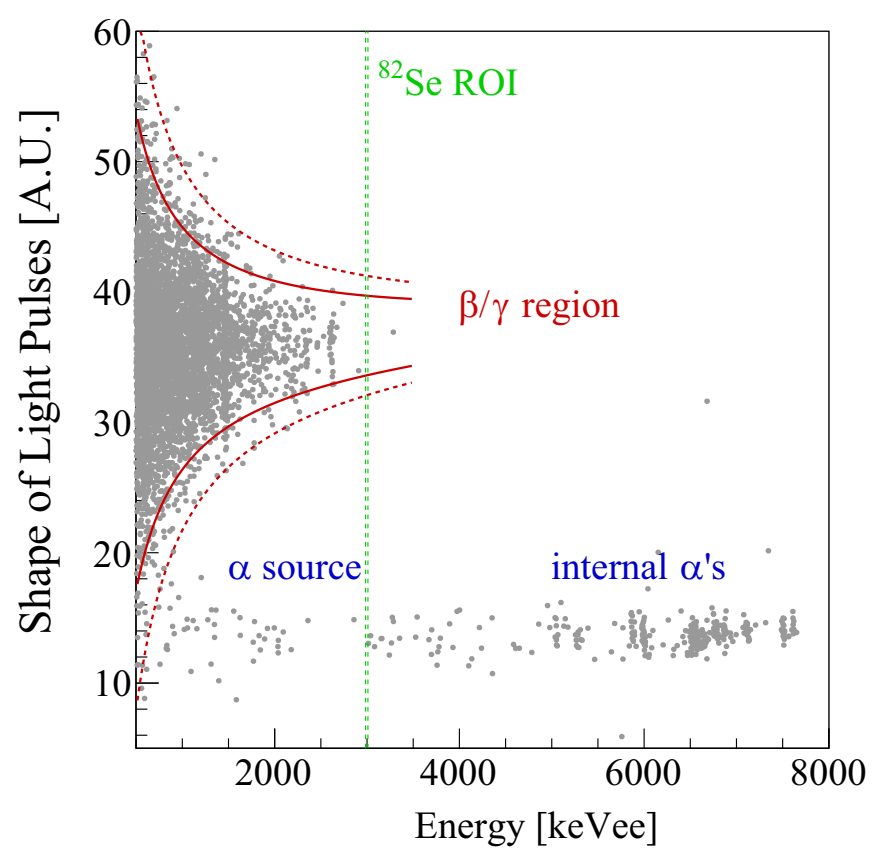

Fig. 4. Shape parameter evaluated on the light pulses acquired by one Ge-LD versus calibrated energy detected on $\mathrm{Zn}^{82}$ Se bolometer. The $\beta / \gamma$ interactions are well separated by the $\alpha$ ones. The $\alpha$ s below $2.5 \mathrm{MeV}$ result from the smeared $\alpha$ source of ${ }^{147} \mathrm{Sm}$, the ones above come from crystal bulk contaminations in ${ }^{232} \mathrm{Th}$, ${ }^{238} \mathrm{U}$ and their daughters. The green band represents the energy region where the signal from $0 \nu \beta \beta$ is expected; the two red continuous and the two dotted boundaries correspond to the exclusion bands to define the signal region respectively at $\pm 2 \sigma$ and $\pm 3 \sigma$ and show the potential of $\alpha$ background rejection capability. No physics model was used to determine the shape of these bands. (For interpretation of the references to color in this figure legend, the reader is referred to the web version of this article.)

two Gaussian functions. We obtained $\sigma=70 \pm 8 \mathrm{eV}$; compatible values were evaluated fitting the ${ }^{55} \mathrm{Fe}$ spectra acquired by the remaining three Ge-LDs.

Since the $\mathrm{Zn}^{82} \mathrm{Se}$ are operated as scintillating bolometers, the $\beta \mid \gamma$ particles interacting within them produced a different LY with respect to the one produced by the $\alpha$ s. As discussed in Ref. [17] the background rejection capability exploiting the LY is effective. Nevertheless, there is a considerable number of $\alpha$ s in which a certain amount of light is lost. This behavior, likely due to surface effects, constitutes a non-negligible and hard to estimate background source. Therefore, we use a more reliable parameter for the particle discrimination. The time development of the light signal shows a strong dependence on the type of interacting particle. As shown in Fig. 3 the light emitted by $\beta / \gamma$ interactions exhibits a slower decay time with respect to the $\alpha$ particles one.

We evaluate a shape parameter on the acquired light pulses, as the one presented in Ref. [18], in order to quantify the difference in the decay time. The results are shown in Fig. 4 where the data collected in about 530 hours of data taking are plotted. On the Y-axis the shape parameter evaluated on the light pulses is reported as function of the energy detected by the $\mathrm{Zn}^{82} \mathrm{Se}-01$ bolometer (X-axis). The two populations ascribed to $\beta / \gamma$ and $\alpha$ interactions can be easily identified: a huge capability to discriminate $\beta / \gamma$ interactions from $\alpha$ ones at the $0 \nu \beta \beta$ energy was found. In the $\alpha$-band we can recognize the smeared $\alpha \mathrm{s}$, produced by the Samarium source (below $2.5 \mathrm{MeV}$ ) and the ones above, produced by crystal bulk contaminations in ${ }^{232} \mathrm{Th},{ }^{238} \mathrm{U}$ and their daughters.

\section{Conclusion and future perspectives}

We tested for the first time three enriched $\mathrm{Zn}^{82}$ Se crystals working as scintillating bolometers. The performances in terms of energy resolution and particle identification are excellent. This test represents the first step in the realization of the final detector CUPID-0, that will be able to demonstrate the potential of this technique for a future tonne-scale experiment with the potential to explore completely the inverted hierarchy region of neutrino masses.

\section{Acknowledgments}

This work was supported by the LUCIFER experiment, funded by ERC under the European Union Seventh Framework Programme (FP7/2007-2013)/ERC Grant agreement no. 247115, funded within the ASPERA 2nd Common Call for R\&D Activities and INFN.

\section{References}

[1] D.R. Artusa, et al., Searching for neutrinoless double-beta decay of ${ }^{130} \mathrm{Te}$ with CUORE, Adv. High. Energy Phys. 2015 (2015) 879871, http://dx.doi.org/10.1155/ 2015/879871, arXiv:1402.6072.

[2] G. Audi, A. Whapstra, C. Thibault, The AME-2003 atomic mass evaluation, Nucl. Phys. A. 729 (2003) 337-676, http://dx.doi.org/10.1016/j. nuclphysa.2003.11.003.

[3] G. Wang, et al., CUPID: CUORE (Cryogenic Underground Observatory for Rare Events) Upgrade with Particle IDentification arXiv:1504.03599.

[4] D.L. Lincoln, J.D. Holt, G. Bollen, M. Brodeur, S. Bustabad, J. Engel, S.J. Novario M. Redshaw, R. Ringle, S. Schwarz, First direct double-? decay Q-Value measurement of ${ }^{82} \mathrm{Se}$ in support of understanding the nature of the Neutrino, Phys. Rev. Lett. 110 (1) (2013) 012501, http://dx.doi.org/10.1103/PhysRevLett.110.012501, arXiv:1211.5659.

[5] S. Pirro, et al., Scintillating double beta decay bolometers, Phys. At. Nucl. 69 (2006) 2109.

[6] J. Beeman, F. Bellini, N. Casali, L. Cardani, I. Dafinei, et al., Characterization of bolometric light detectors for rare event searches, JINST 8 (2013) P07021, http: //dx.doi.org/10.1088/1748-0221/8/07/P07021, arXiv:1304.6289.

[7] J. Beeman, F. Bellini, P. Benetti, L. Cardani, N. Casali, et al., Current status and future perspectives of the lucifer experiment, Adv. High. Energy Phys. 2013 (2013) 237973, http://dx.doi.org/10.1155/2013/237973.

[8] E. Andreotti, et al., ${ }^{130}$ Te neutrinoless double-beta decay with cuoricino, Atropart. Phys. 34 (2011) 822-831.

[9] K. Alfonso, et al., Search for Neutrinoless Double-Beta Decay of ${ }^{130} \mathrm{Te}$ with 
CUORE-0, Phys. Rev. Lett. 115 (10) (2015) 102502, http://dx.doi.org/10.1103/ PhysRevLett.115.102502, arXiv:1504.02454.

[10] M. Clemenza, C. Maiano, L. Pattavina, E. Previtali, Radon-induced surface contaminations in low background experiments, Eur. Phys. J. C 71 (2011) 1805, http://dx.doi.org/10.1140/epjc/s10052-011-1805-0.

[11] S. Pirro, Further developments in mechanical decoupling of large thermal detectors, Nucl. Instrum. Methods A 559 (2006) 672-674, http://dx.doi.org/ 10.1016/j.nima.2005.12.197.

[12] C. Arnaboldi, et al., The front-end readout for CUORICINO, an array of macrobolometers and MIBETA, an array of mu-bolometers, Nucl. Instrum. Methods A 520 (2004) 578.

[13] C. Arnaboldi, G. Pessina, S. Pirro, The cold preamplifier set-up of CUORICINO towards 1000 channels, Nucl. Instrum. Meth. A 559 (2006) 826.
[14] V. Radeka, N. Karlovac, Least-square-error amplitude measurement of pulse signals in presence of noise, Nucl. Instrum. Methods 52 (1967) 86.

[15] E. Gatti, P.F. Manfredi, Processing the signals from solid state detectors in elementary particle physics, Riv. Nuovo Cimento 9 (1986) 1.

[16] G. Piperno, S. Pirro, M. Vignati, Optimizing the energy threshold of light detectors coupled to luminescent bolometers, JINST 6 (2011) P10005, http://dx doi.org/10.1088/1748-0221/6/10/P10005, arXiv:1107.5679.

[17] J. Beeman, F. Bellini, L. Cardani, N. Casali, I. Dafinei, et al., Performances of a large mass ZnSe bolometer to search for rare events, JINST 8 (2013) P05021, http://dx.doi.org/10.1088/1748-0221/8/05/P05021, arXiv:1303.4080.

[18] J.W. Beeman, et al., $\mathrm{ZnMoO}_{4}$ A Promising bolometer for neutrinoless double beta decay searches, Astropart. Phys. 35 (2012) 813-820, http://dx.doi.org/ 10.1016/j.astropartphys.2012.02.013, arXiv:1202.0238. 